\title{
Baryon acoustic oscillations signature in the three-point angular correlation function from the SDSS-DR12 quasar survey
}

\author{
E. de Carvalho ${ }^{1,2 \star}$ A. Bernui, ${ }^{1}$ H. S. Xavier, ${ }^{3}$ C. P. Novaes ${ }^{1,4}$ \\ ${ }^{1}$ Observatório Nacional, Rua General José Cristino 77, São Cristóvão, 20921-400 Rio de Janeiro, RJ, Brazil \\ ${ }^{2}$ Centro de Estudos Superiores de Tabatinga, Universidade do Estado do Amazonas, 69640-000, Tabatinga, AM, Brazil \\ ${ }^{3}$ Instituto de Astronomia, Geofísica e Ciências Atmosféricas, Universidade de São Paulo, Rua do Matão, 1226, 05508-090, São Paulo - SP, Brazil \\ ${ }^{4}$ Instituto de Física, Universidade de São Paulo, Rua do Matão trav. R 187, 05508-090, São Paulo - SP, Brazil
}

5 February 2020

\begin{abstract}
The clustering properties of the Universe at large-scales are currently being probed at various redshifts through several cosmological tracers and with diverse statistical estimators. Here we use the three-point angular correlation function (3PACF) to probe the baryon acoustic oscillation (BAO) features in the quasars catalogue from the twelfth data release of the Sloan Digital Sky Survey, with mean redshift $\bar{z}=2.225$, detecting the BAO imprint with a statistical significance of $2.9 \sigma$, obtained using lognormal mocks. Following a quasi modelindependent approach for the 3PACF, we find the BAO transversal signature for triangles with sides $\theta_{1}=1.0^{\circ}$ and $\theta_{2}=1.5^{\circ}$ and the angle between them of $\alpha=1.59 \pm 0.17 \mathrm{rad}$, a value that corresponds to the angular BAO scale $\theta_{\mathrm{BAO}}=1.82^{\circ} \pm 0.21^{\circ}$, in excellent agreement with the value found in a recent work $\left(\theta_{\mathrm{BAO}}=1.77^{\circ} \pm 0.31^{\circ}\right)$ applying the $2 \mathrm{PACF}$ to similar data. Moreover, we performed two type of tests: one to confirm the robustness of the BAO signal in the 3PACF through random displacements in the dataset, and the other to verify the suitability of our random samples, a null test that in fact does not show any signature that could bias our results.
\end{abstract}

Key words: large-scale structure of Universe - quasars: general - surveys

\section{INTRODUCTION}

Studies of the large-scale structure (LSS) have revealed properties of the Universe which confirm the $\Lambda$ CDM hierarchical scenario for galaxy formation and cosmic evolution (Peacock 1999; Springel et al. 2006; Piattella 2018). The information about LSS has been accessed mainly using the $n$-point correlation function statistics (Peebles 2001; Peebles \& Groth 1975; Groth \& Peebles 1977). Thereby, the two-point correlation function (2PCF) was extensively employed to search for the Baryon Acoustic Oscillations (BAO) imprint in the galaxy and quasar surveys (Peebles \& Yu 1970; Sunyaev \& Zeldovich 1970; Bond \& Efstathiou 1984; Cole et al. 2005; Eisenstein et al. 2005; Paris et al. 2017; Marra \& Isidro 2018). The next order statistics, the three-point correlation function (3PCF), has been used to probe the non-Gaussian features expected in the galaxy distribution (Frieman \& Gaztañaga 1999; Slepian et al. 2017a,b), and to confirm the predictions of non-linear cosmological perturbation theory (see, e.g., Bernardeau et al. 2002, for a review).

The 3PCF is being also used to confirm the BAO features; the first analyses of this type were done by Gaztañaga et al. (2009)

* e-mail: edilsonfilho@on.br with the sixth and seventh data releases (DR) from the Sloan Digital Sky Survey (SDSS), where they found the BAO signature at $\sim 100 \mathrm{Mpc} / h$. Recently, Slepian et al. $(2017 \mathrm{a}, \mathrm{b})$ detected the BAO signal in the 3PCF with $4.5 \sigma$ statistical significance using the SDSS DR12 galaxy sample. Many of the reported works assume a fiducial cosmology to calculate the three-dimensional (3D) comoving distances between the pairs of cosmic objects that form a triangle configuration to finally compute the 3PCF. Some of these works, e.g. Frieman \& Gaztañaga (1999); Jing \& Börner (2004); McBride et al. (2010), perform their analyses in the projected space (Davis $\&$ Peebles 1983). To minimize the impact of redshift distortions, they first calculate the $2 \mathrm{PCF}$ as a function of two coordinates: the redshift space distance into line-of-sight, $\pi$, and projected separation, $r_{p}$, such that $\left(\pi^{2}+r_{p}^{2}\right)^{1 / 2}$ is the observational distance in redshift space. Because the anisotropic redshift space distortion is primarily contained in the $\pi$ coordinate, they integrate along this coordinate resulting in the projected $2 \mathrm{PCF}$, and then the projected 3PCF is obtained through analogous definitions (see, e.g., McBride et al. 2010, for a review).

In 2011, Sánchez et al. (2011) proposed an approach to calculate the two-point angular correlation function (2PACF) in a quasi model-independent way. This methodology was then applied by Carnero et al. (2012) to study the angular BAO signature of the 
DR7 SDSS sample of luminous red galaxies. After that, the 2PACF has been applied to several datasets to investigate the BAO signal at different redshifts (Carvalho et al. 2016, 2017; Salazar-Albornoz et al. 2017; Abbott et al. 2017; Crocce et al. 2017; de Carvalho et al. 2018). Here, we extend to the three-point statistics the approach proposed by Sánchez et al. (2011) and perform for the first time analyses of the three-point angular correlation function (3PACF) based only in the sky angular separation of SDSS quasars located in a thin redshift shell, with mean redshift $\bar{z}=2.225$. We successfully confirm the BAO transversal signature at the same angular position already found in a recent work analyzing these data with the 2PACF (de Carvalho et al. 2018); from now on this reference is termed EdC18.

The main motivations to perform two-dimensional (2D) BAO analyses, instead of the 3D approach, are the following. Differently from the $3 \mathrm{D}$ case where one needs to assume a fiducial cosmology to calculate the comoving distances between pairs of objects in order to construct the $2 \mathrm{PCF}$, in 2D analyses one only uses the angular coordinates, given by the survey catalogue, to calculate angular distances between pairs to search for the BAO features in the 2PACF and 3PACF. An advantage of such model-independent approach is that their results can be combined with other model-independent (or weakly model-dependent) data to impose restrictions on cosmological models or parameters, or simply to compare results obtained in a 3D approach. One can also perform 2D analyses in several noncorrelated thin redshift bins to obtain the best-fit angular diameter distance $D_{A}\left(z ; r_{s}\right)$, to be used in cosmological model or parameter analyses as done by de Carvalho et al. (2018); Carvalho et al. (2016); Carnero et al. (2012); Sánchez et al. (2011). In addition, if the main target in BAO analyses is a statistically significant measurement of the BAO signature, another advantage is that some undesired phenomena that affects such measure in $3 \mathrm{D}$ are minimal or negligible in 2D analyses considering data in thin redshift bins (e.g. the redshift space distortions).

We organise this work as follows. Section 2 gives the details of the quasars, the random, and the mock catalogues employed in the analyses; and the angular correlation function estimators applied to these datasets are presented in section 3 . The data analyses and results are discussed in section 4, while in section 5 we summarise our conclusions.

\section{THE DATA, RANDOM, AND MOCK CATALOGUES}

\subsection{The quasars and randoms dataset}

The data used is part of the twelfth public Data Release Quasar catalogue (DR12Q), from the SDSS-III (Eisenstein et al. 2011) ${ }^{1}$. The DR12Q sample contains 297, 301 quasars from the Baryon Oscillation Spectroscopic Survey (BOSS; Dawson et al. 2013), among which 184,101 have $z \geq 2.15$, covering a total sky area of $9,376 \mathrm{deg}^{2}$. The full sample has been spectroscopically confirmed based on a visual inspection of the spectra of each quasar. The SDSS-III/BOSS limiting magnitudes for quasar target selection are $r \leq 21.85$ or $g \leq 22$ (Paris et al. 2017). The main challenge faced in the quasar BOSS survey was to obtain a high number density sample, satisfying the proposed minimum threshold of 15 quasars per square degree (Paris et al. 2017). This sample is dense enough to perform 2D analyses in thin redshift bins.
In EdC18 we performed a detailed evaluation of the signal-tonoise ratio to select the quasars data for BAO analyses. As a result, we selected a sample of quasars in the thin shell $z \in[2.20,2.25]$, with width $\delta z=0.05$, containing a total of 13,980 quasars distributed between the north and south Galactic hemispheres (in EdC18 we consider only the data in the north Galactic region). The number density of this dataset is large enough to measure the angular BAO signature with a good statistical significance using the 3PACF.

The random catalogues are used to extract the BAO features from the data, for this they must share common properties as those observed in the quasar catalogue. The random samples were generated according to the procedure described in $\mathrm{EdC} 18$; for the present analyses we produced 150 random sets with equal number of objects, homogeneously distributed in the same sky region as the quasars catalogue; 50 of these sets were used for the 2-point and 3point correlation functions statistics, while we employed the other 100 sets for the null test analyses.

\subsection{The Mocks}

The mock quasar catalogues used in this work are full-sky lognormal realizations created with the FLASK $\operatorname{code}^{2}$ (Xavier et al. 2016). To generate such mocks in a single redshift shell, we provided as input: the expected projected number density of quasars of $1.49 \mathrm{deg}^{-2}$ (the same as in BOSS data); and a fiducial angular power spectrum $C_{\ell}$ computed with CAMBsources ${ }^{3}$ (Challinor \& Lewis 2011) for a top-hat redshift bin $(2.20<z<2.25)$, assuming a quasar linear bias of 4.25 , the $\Lambda \mathrm{CDM}$ cosmological parameters measured by Planck (Ade et al. 2016) and a minimal Neutrino contribution (effective number of neutrinos $N_{\text {eff }}=3.046$ and sum of masses $\left.\Sigma m_{\nu}=0.06 \mathrm{eV}\right)$. All $C_{\ell}$ contributions available in CAMBsources (e.g. lensing, redshift space distortions and non-linear clustering) were included. The shift parameter $\lambda$ of the lognormal probability distribution $(-\lambda$ is the minimum value attained by the quasar density contrast) was set to 1 .

Once the mean number density, the shift parameter and the angular power spectra are defined in the lognormal model, all other statistical properties are set in accordance, including the 3PACF (Xavier et al. 2016). We adopted an angular resolution for the mocks of $\sim 0.06 \mathrm{deg}$, set by the Healpix ${ }^{4}$ (Górski et al. 2005) parameter $N_{\text {side }}=1,024$. On scales smaller than this, the mock quasars are distributed homogeneously (their distribution inside a pixel is random). In conformity with the simulation's resolution, we band-limited the realizations to $\ell_{\max }=2,560$. A total of 200 full-sky mock catalogues were produced for our analyses.

\section{THE ANGULAR CORRELATION FUNCTIONS}

Many BAO analyses assume a fiducial cosmology to compute the comoving distance among pairs, then the characteristic scale is found through the $2 \mathrm{PCF}$, and similarly for the computation of the 3PCF. We are interested in the transversal BAO signal, for this we use the angular version of this estimator, i.e., the two-point angular correlation function (2PACF) and the next order, the 3PACF, that will be applied to the quasars data in a thin redshift shell.

\footnotetext{
2 http://www.astro.iag.usp.br/ \{\}flask

3 http://camb.info/sources

4 http://healpix.sourceforge. net
} 


\subsection{The three-point angular correlation function}

The $3 \mathrm{PCF}$ is a complementary tool to characterize the clustering of cosmological tracers like galaxies, quasars, etc. For other applications of the 3PCF see, e.g., Fry \& Seldner (1982); Jing \& Börner (2004); Gaztañaga et al. (2005b); Kulkarni et al. (2007); McBride et al. (2010); Marín (2011); Marín et al. (2013); for alternative statistical tools and clustering analyses see, e.g., Novaes et al. (2014, 2016, 2018); Marques et al. (2018, 2019). Basically, the 3PCF compares the number of triplets of cosmic objects from a dataset that form a triangle configuration, to be called $D D D$, with respect to the number of triplets from a simulated random set of data, termed $R R R$.

Let us start briefly reviewing the basics of the 2PCF. This statistical tool measures the excess probability over a random dataset of finding pairs of cosmic objects from a given catalog. It has been used for many applications in astrophysical problems (Peebles 2001; Bernui et al. 2008; Salazar-Albornoz et al. 2014; Avila et al. 2018 , 2019). To calculate the 2 PCF, the widely used estimator is the Landy-Szalay (LS) estimator (Landy \& Szalay 1993), which has a better performance when compared with other estimators (Kersher et al. 2000) because it results in the smallest deviations for a given cumulative probability, besides having minimal variance and no bias. The LS estimator is defined by

$$
\xi(s) \equiv \frac{D D(s)-2 D R(s)+R R(s)}{R R(s)},
$$

where $D D(s), R R(s), D R(s)$ are the normalized pair counts between data-data, random-random and data-random objects, respectively, where the pairs are separated by the comoving distance $s$ (Landy \& Szalay 1993; Sánchez et al. 2011). In addition, to estimate the 3PCF we consider the Szapudi-Szalay, SS, estimator (Szapudi-Szalay 1998), which is a general extension for all $n$ point correlation functions in 3D. For the case of the 3PCF, $n=3$, the SS estimator assumes the form

$\zeta(S) \equiv \frac{D D D(S)-3 D D R(S)+3 D R R(S)-R R R(S)}{R R R(S)}$,

where the $D D D$ and the other terms are all normalized triplet counts such that three cosmic objects form a triangle of sides given by the triplet $S=\left\{s_{12}, s_{23}, s_{31}\right\}$, where $s_{12}$ is the comoving distance between the objects 1 and 2 and so on (see Marín 2011).

The correlation functions that explore the clustering of objects in the 3D space need to assume a fiducial cosmological model to calculate the 3D distances first and then the comoving distance $s$ between pairs of cosmic objects. However, using the angular version, that is the 2PACF and 3PACF, one can minimize this model dependence, by considering just angular distances in the transversal plane (actually, a thin shell) to the line of sight. In this case, the data is located in a thin redshift bin, and the 2PACF measures the transversal BAO signature. The angular version of the LS estimator, $w(\theta)$, for data in a thin redshift bin with mean redshift $\bar{z}$, is given by (see, e.g., Sánchez et al. 2011; Carnero et al. 2012; Carvalho et al. 2016)

$$
w(\theta) \equiv \frac{D D(\theta)-2 D R(\theta)+R R(\theta)}{R R(\theta)},
$$

where $\theta$ is the angular separation between any pair in the data and/or in the random sample. Analogously, the angular version of the SS estimator (Peebles \& Groth 1975; Frieman \& Gaztañaga 1999; Materne 1988; Cárdenas et al. 2017) for the 3PACF involves
3 variables that define the triangle formed by 3 cosmic objects, is

$W(\Theta) \equiv \frac{D D D(\Theta)-3 D D R(\Theta)+3 D R R(\Theta)-R R R(\Theta)}{R R R(\Theta)}$,

where $\Theta$ represents the triplet of angular distances $\left\{\theta_{1}, \theta_{2}, \theta_{3}\right\}$ of the triangle. Specifically, $\theta_{1}\left(\theta_{2}, \theta_{3}\right)$ is the angular distance between the cosmic objects number $2(3,1)$ and number $3(1,2)$.

One can introduce the reduced 3PACF, defined by Groth \& Peebles (1977) as

$$
q(\Theta)=\frac{W(\Theta)}{w_{1} w_{2}+w_{2} w_{3}+w_{1} w_{3}},
$$

where $w_{i} \equiv w\left(\theta_{i}\right), i=1,2,3$, with $\theta_{i}$ as explained above. According to de Carvalho et al. (2018), the angular BAO scale is $1.77^{\circ} \pm 0.31^{\circ}$ for this redshift bin $z \in[2.20,2.25]$, therefore we have an expectation for the triangle configuration and its scale. Furthermore, we choose to analyze $q$ it instead of $W$ because, as noted by Marín et al. (2013), it appears to be more suitable to study the shape dependence of matter clustering. Besides this advantage, Fry (1994) have shown that the non-linear bias affects just the amplitude of the reduced 3PACF but not the triangle shape considered in the analysis (see also Zheng 2004; Gaztañaga et al. 2009).

Additionally, one can parametrize the triplet configurations in the following way. One first fixes the values $\theta_{1}$ (the angular distance between objects 2 and 3 ) and $\theta_{2}$ (the angular distance between objects 3 and 1$)$, and then calculates the function $q(\Theta)=q\left(\alpha\left[\theta_{3}\right]\right)$, for $\alpha \in\left[0^{\circ}, 180^{\circ}\right]$, which is the angle formed by the sides $2-3$ and 3-1 of the triangle 1-2-3 (the Figure 1 from Gaztañaga et al. 2005b, illustrates the meaning of $\alpha$ ):

$$
\cos \alpha=\frac{\theta_{1}^{2}+\theta_{2}^{2}-\theta_{3}^{2}}{2 \theta_{1} \theta_{2}} .
$$

For $\alpha=0^{\circ}$ the configuration is termed collapsed triangle and the size of the third side of the triangle is $\theta_{3}=\left|\theta_{2}-\theta_{1}\right|$. For the case $\alpha=180^{\circ}$, termed elongated triangle, the third side is $\theta_{3}=$ $\theta_{2}+\theta_{1}$ (Gaztañaga \& Scoccimarro 2005a; McBride et al. 2010).

To find the angular scale of the BAO bump in the reduced 3PACF, $q(\alpha)$, we follow the approach of Sánchez et al. (2011) based on an empirical parametrization of $q(\alpha)$ which consists of a quadratic function to describe the overall shape, in some works called the 'U'-form, plus a Gaussian function to describe the BAO bump

$$
q(\alpha)=m+n \alpha+p \alpha^{2}+C \exp ^{-\left(\alpha-\alpha_{\mathrm{FIT}}\right)^{2} / 2 \sigma_{\mathrm{FIT}}^{2}},
$$

where $m, n, p, C, \alpha_{\mathrm{FIT}}$, and $\sigma_{\mathrm{FIT}}$ are free parameters. The best-fit of the reduced 3PACF obtained with this expression provides $C$, $\alpha$ FIT and $\sigma_{\text {FIT }}$ which describes the BAO signal shape. The parameters $m, n$, and $p$ control both the amplitude and the form of the parabola.

\section{DATA ANALYSES AND RESULTS}

In this section, we perform the analyses that lead us to a robust measurement of the angular BAO scale in the DR12 SDSS quasar catalog through the reduced 3PACF statistic, $q(\alpha)$. This work extends the analyses done in EdC18, where the 2PACF was applied to the north Galactic hemisphere data of the DR12 quasar catalog, in the same redshift bin as here, finding a BAO signal at $1.77^{\circ} \pm 0.31^{\circ}$ with statistical significance of $2.12 \sigma$. 


\subsection{The reduced 3PACF results}

Now we shall compute the reduced 3PACF, $q(\Theta)$, given in equation (5), but before we need to calculate the functions $W(\Theta)$ and $w_{i}$ for $i=1,2,3$, using the equations (3) and (4) for triangle configurations with fixed $\theta_{1}=1.0^{\circ}$ and $\theta_{2}=1.5^{\circ}$. To compute these functions we use 50 random samples, generated according to the procedure described in EdC18. In this way, we obtain the 3-point angular correlations for our quasars catalogue, in the redshift range $z \in[2.20,2.25]$, with mean redshift $\bar{z}=2.225$, as shown in the Figure 1 .

The procedure for computing the reduced $3 \mathrm{PACF}$ consists on calculating the $2 \mathrm{PACF}$ and $3 \mathrm{PACF}$ of the quasar catalogue using each random sample. The final results are obtained as the mean over the 50 sets of each $w(\theta)$ and $W(\alpha)$ data points for every $\theta$ and $\alpha / \pi$ bins. Finally, we used the equation (5) to obtain the reduced 3PACF. The error bars shown in Figure 1 were obtained from the quasar mocks' covariance matrix (estimated according to Sec. 4.2) as the square root of the main diagonal for each function, namely $w(\theta), W(\alpha)$, and $q(\alpha)$ (see Figure 2).

The binning choice, mainly for the 3PACF case, is a compromise that has a strong impact in the signal to noise ratio (see Marín 2011, and references therein). Besides that, triplet configurations depend on the angular separation $\theta$ between pairs and, to form a reasonable number of triplets, one must choose a value for $\Delta \theta$, which will define the resolution of the results, in such a way that we actually do not have exact values of $\theta_{1}$ and $\theta_{2}$, but bins of $\theta_{1} \pm \Delta \theta$ and $\theta_{2} \pm \Delta \theta$. A low resolution implies a small number of triplets by bin and a small signal-to-noise ratio. For our analyses, after several tests, we have chosen $\Delta \theta=0.15^{\circ}$ which allow us to find a significant number of triplet configurations providing a $\mathrm{BAO}$ signature with a good statistical significance, as we shall see.

Note that the reduced 3PACF was estimated for equally spaced bins of $\alpha / \pi$ in the range $0.0 \leq \alpha / \pi \leq 1.0$, in a total of $N_{b}=10$ bins. Then, to extract the BAO features we fit equation (7) to the reduced 3PACF data using the covariance matrix obtained from quasar mocks (see Figure 2). The BAO bump is identified at the position $\alpha_{\mathrm{FIT}}=1.57 \pm 0.081_{(\text {stat })}$ rad or $\alpha_{\mathrm{FIT}}=89.89^{\circ} \pm 4.6_{(\text {stat })}^{\circ}$.

The statistical error, denoted stat, was obtained in the following way. We produce 10,000 synthetic $q(\alpha)$ datasets and extract the U-form and BAO bump parameters by fitting them according to the empirical parametrization $q(\alpha)$, given by equation (7). Each synthetic dataset was generated by setting the measured $q(\alpha)$ as the true one and adding to it Gaussian random errors according to the measured covariance matrix. Figure 3 shows the histogram of the recovered BAO bumps, $\alpha_{\mathrm{FIT}}$, from these 10,000 synthetic realisations, whose standard deviation, $\sigma_{\text {stat }}=0.081 \mathrm{rad}$, gives a measure of the statistical uncertainty in our procedure.

The systematic error, denoted sys, will be calculated in detail in another section. According to the values considered for $\theta_{1}, \theta_{2}$ above and using the equation (6) the angle $\alpha=\alpha_{\text {FIT }}$ corresponds to $\theta_{\text {FIT }} \equiv \theta_{3}=1.80^{\circ}$.

\subsection{The Covariance Matrix estimation}

To estimate the covariance matrix and the significance of our results we have used a sample with $N=200$ quasar mocks described above (see the subsection 2.2). For each mock, we extract the information about the $2 \mathrm{PACF}$ and the $3 \mathrm{PACF}$ and finally calculate the reduced 3PACF, $q(\alpha)$. The covariance matrix for $w(\theta), W(\Theta)$ and $q(\Theta)$ was estimated using the following expression (see Gaztañaga

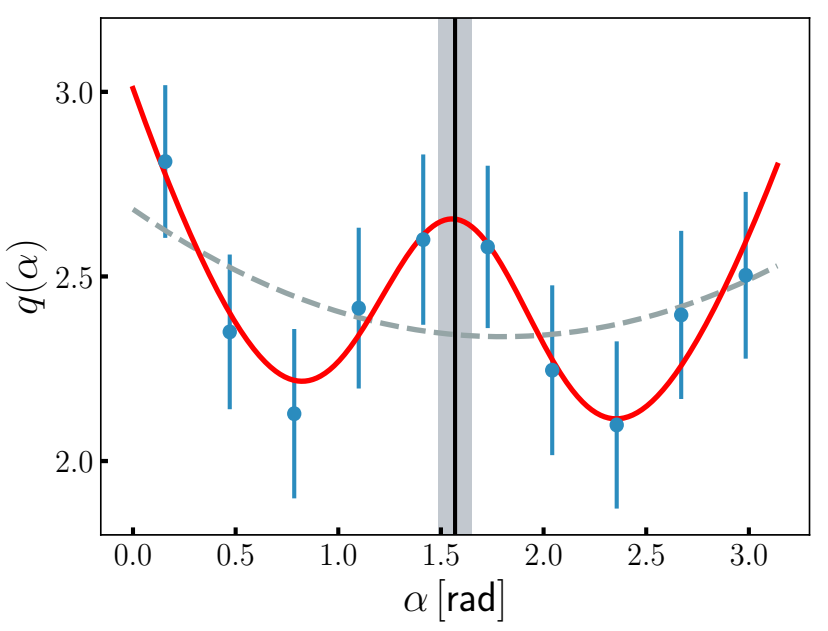

Figure 1. The reduced 3PACF (dots), $q(\alpha)$, calculated from the quasars sample DR12 - SDSS with $\bar{z}=2.225$, for fixed values $\theta_{1}=1.0^{\circ}$ and $\theta_{2}=1.5^{\circ}$. The best-fit of these data is $\alpha_{\text {FIT }}=1.57 \pm 0.081_{(\text {stat })}$ rad (vertical line; error bar repesented by the gray region), where the continuous line was obtained using the equation (7) considering $N_{b}=10$ bins. The dashed line corresponds to the best-fit curve for the non-BAO signal case, i.e., $C=0$ in equation (7).

et al. 2009):

$$
\operatorname{Cov}_{i j}=\frac{1}{N} \sum_{k=1}^{N}\left[x_{k}(i)-\hat{x}(i)\right]\left[x_{k}(j)-\hat{x}(j)\right] .
$$

Here, the $x_{k}(i)$ represents the statistic used [i.e., $w(\theta), W(\Theta)$, or $q(\Theta)]$ in the bin $i$ for each mock $k$, and the $\hat{x}(i)$ is the mean value for this statistic over the 200 mock samples in that bin.

The error of $x(i)$ is the square root of the main diagonal, $\delta x(i)=\sqrt{\operatorname{Cov}_{i i}}$. We show the covariance matrix in Figure 2 for the case of the reduced 3PACF, $q(\alpha)$.

The statistical significance of the BAO signal measurement is obtained through the $\chi^{2}$ methodology, using the inverse of the covariance matrix as

$$
\chi^{2}(\alpha)=\left[q(\alpha)-q^{\mathrm{FIT}}(\alpha)\right]^{T} \operatorname{Cov}^{-1}\left[q(\alpha)-q^{\mathrm{FIT}}(\alpha)\right] .
$$

The symbols [] and [ $]^{T}$ represent column vectors and row vectors, respectively. We adjusted the parameters of equation (7), based in the minimum $\chi^{2}$ method, for two cases: considering $C$ as free parameter, $C \neq 0\left(\chi_{\min }^{2}=2.24\right)$, and imposing $C=0$ $\left(\chi_{\min }^{2}=16.00\right)$, the latter representing the non-BAO case (see Figure 1). Table 1 shows the best-fit parameters for each case.

As a result, the best-fit of the non-BAO case (7 degrees of freedom, dof), compared to the BAO case ( $4 d o f$ ), is disfavoured by $\Delta \chi^{2}=13.76(7-4=3$ dof $)$. Therefore, our detection of the BAO signal has a significance of $2.9 \sigma$.

It is worth to notice that this statistical significance depends on the covariance matrix derived from the lognormal simulations, which could underestimate the error bars and, consequently, overestimate the statistical significance. As a comparison, we have used the Jackknife approach to extract the covariance matrix and finding a significance of $1.95 \sigma$. However, Norberg et al. (2009) have 


\begin{tabular}{ccc}
\hline Parameters & Eq. $7(C=0)$ & Eq. $7(C \neq 0)$ \\
\hline$m$ & $2.68 \pm 0.31$ & $3.01 \pm 0.19$ \\
$n$ & $-0.38 \pm 0.30$ & $-1.56 \pm 0.54$ \\
$p$ & $0.11 \pm 0.10$ & $0.48 \pm 0.17$ \\
$C$ & 0.0 & $0.93 \pm 0.30$ \\
$\sigma_{F I T}$ & - & $0.41 \pm 0.12$ \\
$\alpha_{F I T}$ & - & $1.57 \pm 0.081$ (stat) \\
\hline
\end{tabular}

Table 1. The best-fit parameters of equation (7), for the BAO $(C \neq 0)$ and non-BAO $(C=0)$ cases (see Figure 1$)$, obtained through the $\chi^{2}$ statistics, equation (9), using the covariance matrix shown in Figure 2.

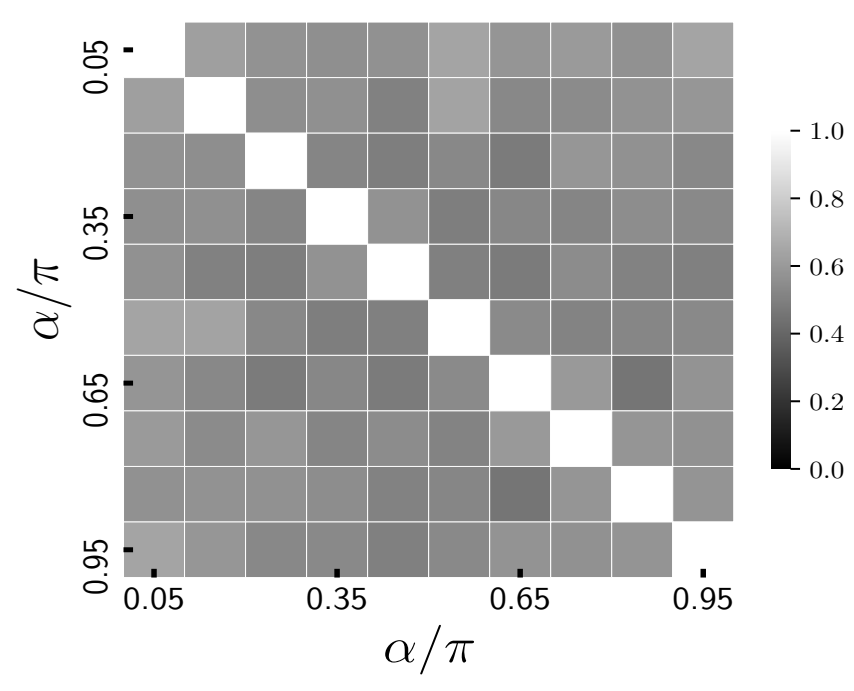

Figure 2. The correlation matrix for the reduced 3PACF, $q(\alpha)$, obtained from 200 quasar mocks (see section 2 for details of how these mocks were produced, and subsection 4.2 for the matrix calculation).

shown that the Jackknife approach could overestimate the error bars, underestimating the statistical significance.

\subsection{Spectroscopic- $z$ Error}

As shown by Sánchez et al. (2011), the primary source of systematic errors in the $2 \mathrm{PACF}$ and, consequently, in the 3PACF, comes from the uncertainty in the measurement of the redshift, $z$, particularly large in the case of photometric redshift surveys with broadband filters as the DES survey ${ }^{5}$. On the other hand, narrow-band filters from current (Eriksen et al. 2019) and forthcoming (Benitez et al. 2014) surveys deal with photo- $z$ errors that are competitive with spectro- $z$ errors.

In the case of the sample studied here, the DR12Q catalogue from the SDSS, the data is spectroscopic and the estimation of $z$ is very precise, as described by Paris et al. (2017). The error associated to spectroscopic measurements is $\delta z=0.003$ (a $3 \sigma$ error; Laurent et al. 2016) which we shall call spec- $z$ error.

5 https: / / www . darkenergysurvey.org/

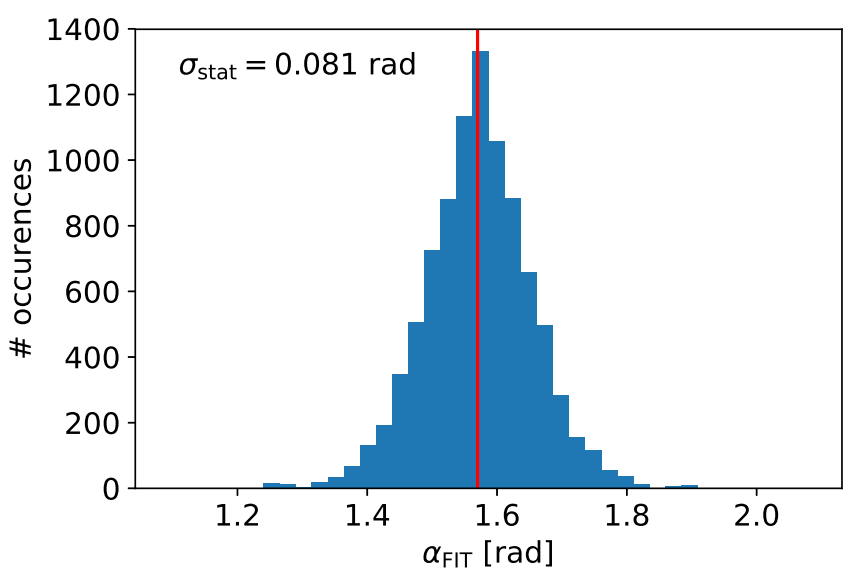

Figure 3. Histogram of best-fitting $\alpha_{\mathrm{FIT}}$ for 10,000 synthetic realisations of $q(\alpha)$, assuming the model given by eq. (7), the parameters given by Table $1(C \neq 0)$ and the Gaussian random errors generated from the covariance matrix estimated from the data. The red vertical line shows the true value, and the standard deviation of the recovered $\alpha_{\mathrm{FIT}}$ is $\sigma_{\text {stat }}=0.081 \mathrm{rad}$.

To estimate the impact of a redshift uncertainty, $\delta z$, in our analyses, we shall perform a test. Consider that the redshift values, $z_{i}$, given in the DR12Q catalogue are the correct ones. For each $z_{i}$ we produce a simulated error according to a Gaussian distribution with mean $z_{i}$ and standard deviation $\left(1+z_{i}\right) \delta z$. We applied this methodology to generate 100 spec- $z$ quasar catalogues, where a given quasar appears in each of these catalogues at a different redshift, whose displacements from the correct values follow such Gaussian distribution. In Figure 4 we show the difference between the $\alpha_{\text {FIT }}$ adjusted from the 'true' quasar sample and the one obtained from each simulated spec- $z$ quasar sample. Then, the relative error associated with the spec- $z$ error is $5 \%$ for the reduced $3 \mathrm{PACF}$ case, and $4 \%$ for the $2 \mathrm{PACF}$ case. This means that the systematic error in the 3PACF BAO measurement is $0.08 \mathrm{rad}$ in $\alpha$. Other sources of systematic errors are the redshift space distortions and the projection effects. However, for the sample in study, their contribution is expected to be small (see, e.g., Sánchez et al. 2011).

\subsection{Robustness of BAO signal and the Null test}

As observed by Gaztañaga et al. (2009), a robustness test of the analyses in $n$-point correlation studies is made by confirming the $\mathrm{BAO}$ signature in the $2 \mathrm{PACF}$ and $3 \mathrm{PACF}$ individually. Once the signal is detected in both statistics one can consider that the BAO detection is robust. In our case, we have obtained the $\mathrm{BAO}$ signature using the 2PACF in EdC18, and in the present analyses we confirm it, with a good statistical significance, with the $3 \mathrm{PACF}$.

We also perform the null test to investigate the behaviour of the $2 \mathrm{PACF}$ and $3 \mathrm{PACF}$ estimators when the data is replaced by a random catalogue; this procedure is repeated with several random catalogues to compute the average. For this, we generated 100 extra random samples (see section 2), to replace the data 100 times, and for each one we obtained the $2 \mathrm{PACF}$ and $3 \mathrm{PACF}$, finally calculating the mean $2 \mathrm{PACF}$ and the mean $3 \mathrm{PACF}$. This procedure follows the same methodology as described in EdC18. The results are shown in the Figure 5, where we present the $n$-point statistics $(n=2,3)$ calculated from the quasars data, that is: the 2PACF, $w(\theta)$, in the upper plot, and the 3PACF, $W(\alpha)$ in the bottom plot, both data represented by dot symbols. In the same panels of Figure 5 we show for 


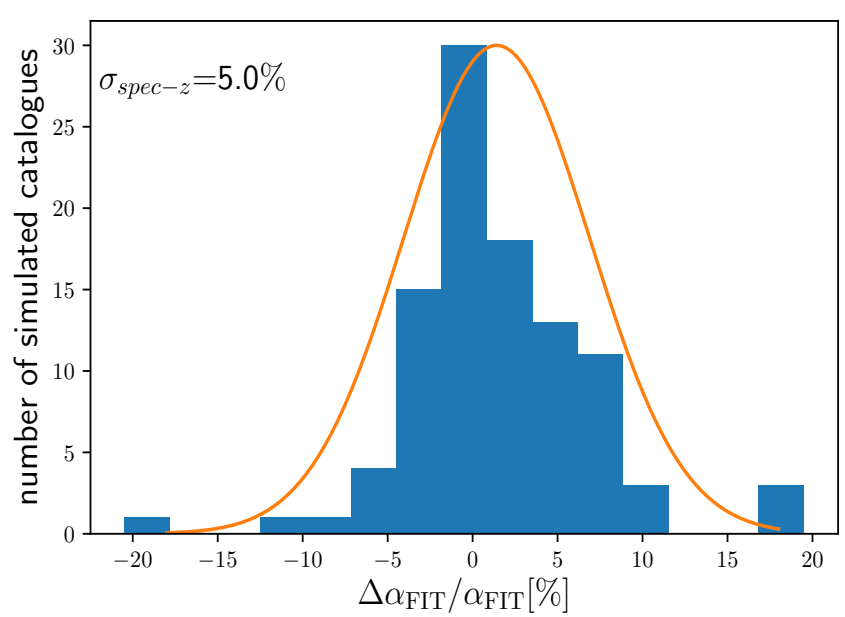

Figure 4. Histogram of the difference $\Delta \alpha_{\text {FIT }}$ between the $\alpha_{\text {FIT }}$ obtained from the quasars data compared to the values obtained from the simulated spec- $z$ samples. This spec- $z$ errors impact the measurements of the BAO signature as a systematic error with a relative amplitude of $5 \%$.

comparison the results from to the null test analyses, represented by square symbols. All error bars are the standard deviation computed from the 100 datasets. As observed, for the null test the $w(\theta)$ and $W(\alpha)$ data points are zero, as expected, confirming that the random samples have no signature that could contaminate our results.

\subsection{Small Shifts Criterium}

This criterium is one more test to validate our results by examining if the signal observed in the 3PACF is not originated by statistical noise, an effect always present in the $n$-point correlation analyses.

To apply this test we have followed the same procedure used in EdC18 (see also Carvalho et al. 2016). We perturb the quasars original positions in the sky according to a Gaussian distribution, in three cases: considering its standard deviation as $\sigma_{s}=0.1^{\circ}, 0.2^{\circ}$, and $0.3^{\circ}$. Geometrically, this process means that the quasars positions are randomly shifted in direction and with displacements of different sizes (following a Gaussian distribution). In Figure 6 we illustrate the effect of this procedure, where even for the intense shake corresponding to $\sigma_{s}=0.2^{\circ}$ or maximum displacement of $1.0^{\circ}$, the BAO signal is highly suppressed but still survives exhibiting the robustness of our result. In the most severe case, $\sigma_{s}=0.3^{\circ}$, a very small $\mathrm{BAO}$ signature could be there, but due to the error bars the result appears compatible with the absence of signal.

\subsection{Projection effect in the 3PACF}

To access the $\theta_{\mathrm{BAO}}$ we need to correct the $\alpha_{\mathrm{FIT}}$ with respect to the projection effect which produces a shift in the BAO bump position (Sánchez et al. 2011). For this, we convert $\zeta(S)$ into $W(\Theta)$ by using the 3PCF result provided by Pertubation Theory (see Fry 1984; Frieman \& Gaztañaga 1999; Barriga \& Gaztañaga 2002; Gaztañaga et al. 2009) and the relation

$$
\begin{array}{r}
W\left(\theta_{12}, \theta_{23}, \theta_{13}\right)=\int d z_{1} \phi\left(z_{1}\right) \int d z_{2} \phi\left(z_{2}\right) \\
\int d z_{3} \phi\left(z_{3}\right) \zeta\left(r_{12}, r_{23}, r_{13} ; \bar{z}\right),
\end{array}
$$

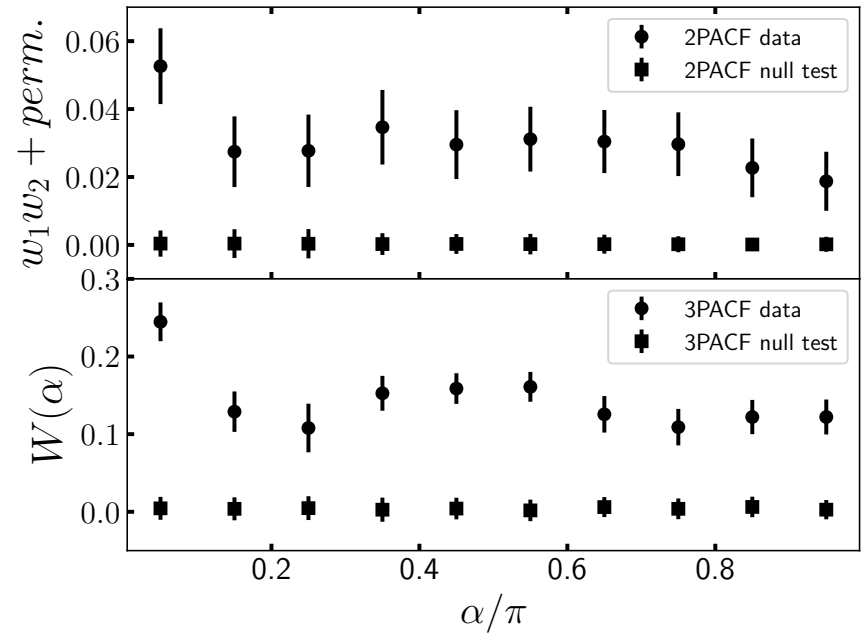

Figure 5. The combination of several $2 \mathrm{PACF} w_{1} w_{2}+w_{1} w_{3}+w_{2} w_{3}$ (upper panel), and the 3PACF (bottom panel), where the data points (circles) correspond to the analyses of the quasars sample DR12. In both panels the data square symbols represent the null test, obtained by replacing the quasars data catalogue with a random catalogue, performing this operation 100 times, and then considering the mean and standard deviation for the data (squares) and error bars, respectively.

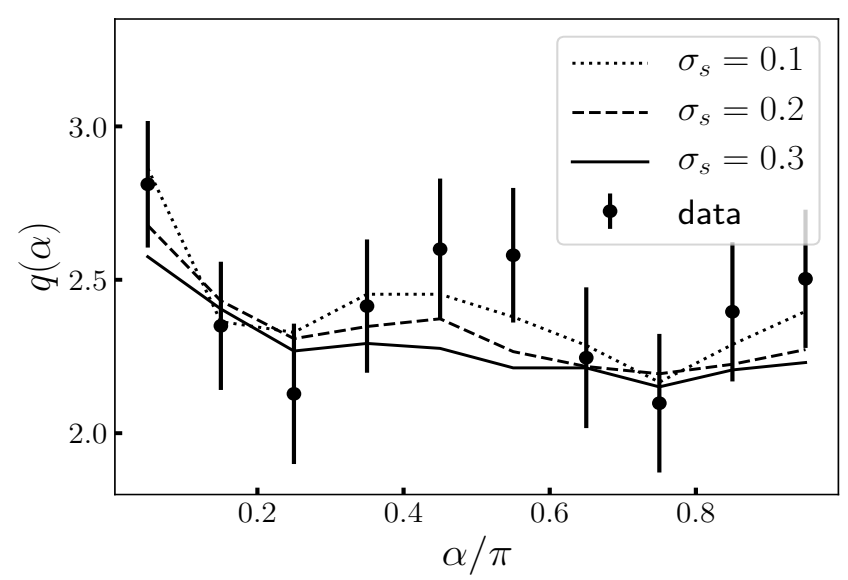

Figure 6. The reduced 3PACF, $q(\alpha)$, for the original quasars catalogue (dots), using the small shift criterium as described in the text (see the subsection 4.5). We have used $\sigma_{s}=0.1^{\circ}, 0.2^{\circ}$, and $0.3^{\circ}$ (dotted, dashed and continuous lines, respectively) to perturb the original quasar positions. As observed, the BAO signature is very robust, clearly appearing still for shifts as intense as a Gaussian shift with $\sigma_{s}=0.2^{\circ}$ (the angular positions of the quasars are shifted at a maximum distance of $1.0^{\circ}$ ).

where $\bar{z}=\left(z_{1}+z_{2}+z_{3}\right) / 3$ and $\phi(z)$ is the redshift selection function normalized to unity within the shell of width $\delta z$. In the case of the $2 \mathrm{PACF}$, we followed the procedure described in Carvalho et al. (2016); de Carvalho et al. (2018). As a reference model we used the $\Lambda \mathrm{CDM}$ model with cosmological parameters from Planck (Aghanim et al. 2018).

To calculate the projection effect for our data with $z \in$ $[2.20,2.25]$, that is, in a shell of width $\delta z=0.05$, we evaluated the above relation in two cases: $\delta z=0.0$ and $\delta z=0.05$, 
and then calculated the relative difference, $\Delta \equiv\left(\alpha_{\mathrm{FIT}} \mid \delta z=0.05-\right.$ $\left.\left.\alpha_{\mathrm{FIT}}\right|_{\delta z=0.0}\right) /\left.\alpha_{\mathrm{FIT}}\right|_{\delta z=0.0}$, in the BAO bump position. Thus, we obtain a relative difference of $\Delta=1.12 \%$. Applying this shift to $\alpha_{\mathrm{BAO}}=(1+\Delta) \alpha_{\mathrm{FIT}}$, we obtain $\alpha_{\mathrm{BAO}}=1.59 \mathrm{rad}$.

\subsection{Validation of the results via fiducial cosmology}

Finally, we shall test the validity of our results assuming a fiducial cosmology. In fact, it is important to evaluate if the use of an empirical parametrization, as given by equation (7), could bias our result. To do this, we use the theoretical realisation of the reduced 3PACF (applying the same procedure described by Barriga \& Gaztañaga 2002), considering as fiducial cosmology the flat $\Lambda \mathrm{CDM}$, with $\left(\Omega_{m}, h, \Omega_{b}, \sigma_{8} . n_{s}\right)=(0.31,0.7,0.059,0.8,0.97)$, and using a non linear power spectrum derived from Perturbation Theory. We follow a local biasing model with (Fry \& Gaztañaga 1993; Frieman \& Gaztañaga 1994; Desjacques et al. 2018)

$$
\delta_{Q}=\sum_{k=0}^{\infty} \frac{b_{k}}{k !} \delta_{m}^{k},
$$

where $\delta_{i}$ is the density contrast, for quasar, $i=Q$, and for matter, $i=m$. This way, in the leading order we have, for the reduced 3PACF, $q_{Q}=\left(1 / b_{1}\right) q_{m}+b_{2} / b_{1}^{2}$, where $b_{1}$ is the usual linear (local) bias, used here as the effective bias parameter $b_{\text {eff }}=4.25$ for quasars (Laurent et al. 2016). Since the non-local bias term contributes only by shifting the $q_{Q}$ curve, with no effect in its shape, we consider $b_{2}=0$ (Frieman \& Gaztañaga 1999).

In addition, following Sánchez et al. (2011); Crocce et al. (2011), we model the selection function as

$$
\phi(z)=\frac{d N_{Q}}{d z} W(z),
$$

where $W(z)$ is the window function encoding our redshift cuts, $z \in$ $[2.20,2.25]$ (i.e., $W=1$ inside the shell and $W=0$ outside the shell). The term $d N_{Q} / d z$ corresponds to the distribution of quasars as a function of the redshift, chosen to be the Gaussian curve bestfitted to the DR12Q distribution, in the range $1.1<z<4.0$. The reduced 3PACF resulting from such theoretical calculation is shown as a continuous line in Figure 7. Notice that this theoretical curve is obtained by fixing the angular distances at $\theta_{1}=1.0^{\circ}$ and $\theta_{2}=1.5^{\circ}$, and the range $\alpha=[0, \pi]$, i.e., the same values used in the data analyses.

Then, to validate the performance of equation (7) in correctly fitting the data, we repeated the same procedure of generating 10,000 synthetic realizations described in section 4.1 , but using the theoretical $q(\alpha)$ (continuous line in Figure 7) as the true one. Figure 8 shows the histogram of the relative difference between the input (true) value $\alpha_{B A O}$ and the $\alpha_{F I T}$ estimates obtained by fitting the equation (7) to each of these realizations (see Figure 7 for an illustrative example of this fitting procedure). The mean value of these differences has a $2.1 \%$ deviation with respect to the input value, which represents an error of $\sigma_{\text {param }}=0.033 \mathrm{rad}$. In fact, this error accounts for only a small fraction of the systematic error, whose main contribution comes from the spectroscopic error. Adding both contributions in quadrature we have $\sigma_{\text {sys }}=0.087$ rad. Finally, we use equation (6) to find $\theta_{\mathrm{BAO}}=1.82^{\circ} \pm 0.21^{\circ}$, considering a combined computation using both the statistical and the systematic errors, as done by Carnero et al. (2012), and the errors in $\theta_{1}$ and $\theta_{2}$.

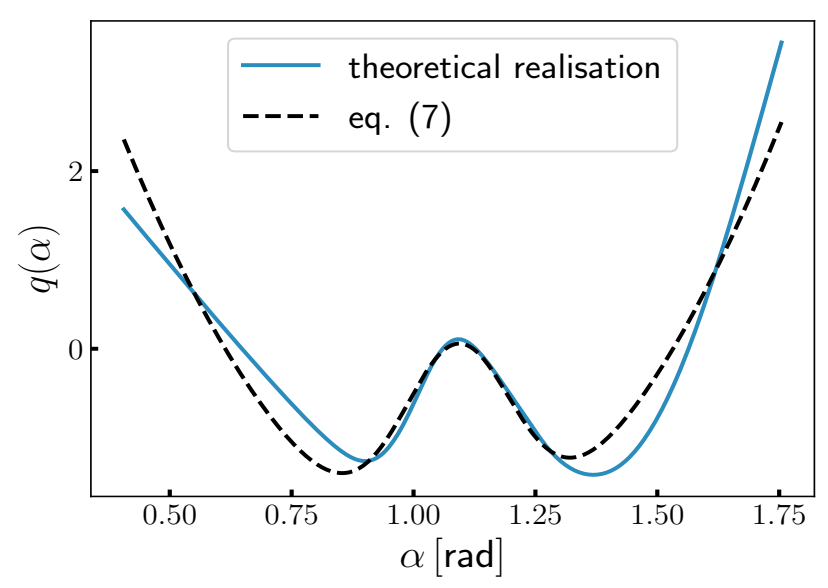

Figure 7. Theoretical calculation of the reduced 3PACF (continuous line) produced according to Barriga \& Gaztañaga (2002) using the CDM power spectrum solution (derived from Perturbation Theory; Fry 1984), including the effective bias parameter for quasars (see text for details). The dashed line represents an illustrative example of the fitting procedure using equation (7). For comparative purposes, we plotted the curves around the BAO bump. The precision and accuracy to recover the BAO signal position was also examined and the results are displayed in the Figure 8.

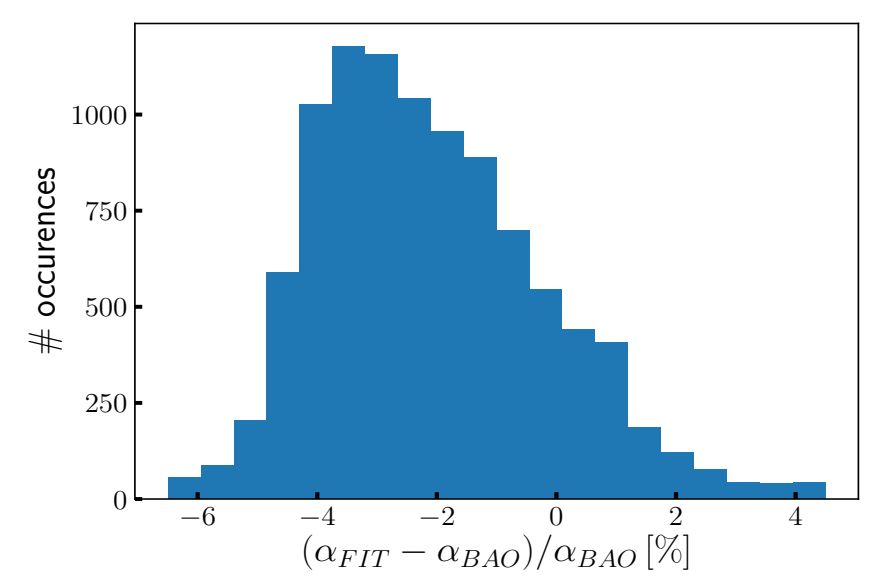

Figure 8. Performance test for recovering the $\alpha_{B A O}$. Histogram of the relative difference between the $\alpha_{B A O}$ (input value) and the best-fitting $\alpha_{F I T}$ value obtained by using the equation ( 7 ) for 10,000 synthetic realisations of $q(\alpha)$. This analysis reveals that the mean value of the differences has a $2.1 \%$ deviation with respect to the input value, which represents an error of $\sigma_{\text {param }}=0.033 \mathrm{rad}$.

\section{CONCLUSIONS AND FINAL REMARKS}

The clustering of matter structures in the Universe is currently probed with large deep surveys by the $n$-point correlation function. In a previous work, EdC18, we used the angular version of the 2-point statistic, the $2 \mathrm{PACF}$, to study the BAO phenomenon in the DR12 quasars catalogue from the SDSS, with $z \in[2.20,2.25]$ considering quasars located in the north Galactic hemisphere, detecting the transversal $\mathrm{BAO}$ signal at $\theta_{\mathrm{BAO}}^{2 \mathrm{PACF}}=1.77^{\circ} \pm 0.31^{\circ}$. 
Here we also studied the BAO features in the SDSS DR12 quasars catalogue, in the same redshift bin as the above analyses but using the angular version of the 3-point statistic, the 3PACF, considering data from the north and south Galactic hemispheres. We detect a transversal $\mathrm{BAO}$ signal with statistical significance, of $2.9 \sigma$, at $\theta_{\mathrm{BAO}}^{3 \mathrm{PACF}}=1.82^{\circ} \pm 0.21^{\circ}$, in excellent agreement with the measurement done with the 2PACF (de Carvalho et al. 2018), successfully confirming this BAO signature for quasars at the mean redshift $\bar{z}=2.225$.

Additionally, we also performed diverse robustness tests to confirm several steps of our procedure to find the BAO signature with the reduced $3 \mathrm{PACF}$ in these quasars data. To estimate the error bars and the significance of our results we have used a sample of 200 quasar mocks. For each mock, we extracted the information about the 2PACF and the 3PACF and finally calculated the reduced 3PACF, $q(\alpha)$. The covariance matrix for each case was estimated using the procedure explained in the subsection 4.2.

The significance of the result was accessed comparing the parametrization given in the equation (7) with and without BAO signal and using the inverse of the covariance matrix coming from the mocks. Finally, the successful result from the null-test guarantees that the random samples have no signature that could contaminate our results.

\section{ACKNOWLEDGEMENTS}

The authors acknowledge insightful discussions with Thiago Pereira and Eusébio Sánchez. EdC acknowledges the PROPGCAPES/FAPEAM program. $\mathrm{AB}, \mathrm{HX}$, and $\mathrm{CN}$ acknowledge the support of the Brazilian agencies CNPq, FAPESP, and FAPERJ, respectively. We also thank the CAPES PVE project 88881.064966/2014-01, within the Science without Borders Program.

\section{REFERENCES}

Abbott T. M. C. et al., 2017, arXiv:1712.06209

Ade P. A. R. et al., 2016, Astronomy \& Astrophysics, 594, A13; arXiv: 1502.01589

Aghanim N. et al., 2018, arXiv:1807.06209

Avila F., Novaes C. P., Bernui A., de Carvalho E., 2018, JCAP 12, 041; arXiv: 1806.04541

Avila F., Novaes C. P., Bernui A., de Carvalho E., Nogueira-Cavalcante J. P., 2019, MNRAS 488, 1481; arXiv: 1906.10744

Barriga J., Gaztañaga E., 2002, MNRAS 333 443; arXiv:astro-ph/0112278

Benitez N. et al., 2014; arXiv:1403.5237

Bernardeau F., Colombi S., Gaztañaga E., Scoccimarro R., 2002, Physics Reports, 367, 1; arXiv:astro-ph/0112551

Bernui A., Ferreira I. S., Wuensche C. A., 2008, Astrophys. J., 673, 968; arXiv:0710.1695

Bond J. R., Efstathiou G., 1984, Astrophys. J., 285, L45

Cárdenas-Montes M. et al., 2017, arXiv:1709.03264

Carnero A. et al., 2012, MNRAS, 419, 1689; arXiv:1104.5426

Carvalho G. C. et al., 2016, Phys. Rev. D, 93, 023530; arXiv:1507.08972

Carvalho G. C. et al., 2017; arXiv:1709.00271

Challinor A., Lewis A., 2011, Phys. Rev. D, 84, 043516; arXiv:1105.5292

Cole S. et al., 2005, MNRAS, 362, 505; arXiv:astro-ph/0501174
Crocce M., Cabre A., Gaztañaga E., 2011, MNRAS, 414, 329; arXiv: $1004.4640 v 2$

Crocce M. et al., 2018, MNRAS, sty2522; arXiv:1712.06211

Davis M., Peebles P. J. E., 1983, Astrophys. J., 267, 465

Dawson K. S., Schlegel D. J., Ahn C. P., et al., 2013, Astronom. J., 145, 10.

de Carvalho E., Bernui A., Carvalho G. C., Novaes C. P., Xavier H. S., 2018, JCAP, 04, 064; arXiv:1709.00113

Desjacques V., Jeong D., Schmidt F., 2005, Physics Reports, 733, 1; arXiv:1611.09787v5

Eisenstein D. J. et al. (SDSS Collaboration), 2005, Astrophys. J., 633, 560; arXiv:astro-ph/0501171

Eisenstein D. J. et al., 2011, Astrophys. J., 142, 72; arXiv:1101.1529

Eriksen M. et al., 2019, MNRAS, 484, 4200; arXiv:1809.04375

Frieman J. A., Gaztañaga E., 1994, Astrophys. J., 425, 392; arXiv:astro$\mathrm{ph} / 9306018 \mathrm{v} 1$

Frieman J. A., Gaztañaga E., 1999, Astrophys. J. Lett., 521, L83; arXiv:astro-ph/9903423

Fry J. N., Seldner M., 1982, Astrophys. J., 259, 474

Fry J. N., 1984, ApJ 279, 499

Fry J. N., Gaztañaga E., 1993, Astrophys. J., 413, 447

Fry J. N., 1994, Phys. Rev. Lett. 73, 215.

Gaztañaga E., Scoccimarro R., 2005, MNRAS, 361, 824; arXiv:astro$\mathrm{ph} / 0501637$

Gaztañaga E., Norberg P., Baugh C. M., Croton D. J., 2005, MNRAS, 364, 620; arXiv:astro-ph/0506249

Gaztañaga E., Cabré A., Castander F., Crocce M., Fosalba P., 2009, MNRAS, 399, 801; arXiv:0807.2448

Górski K. M. et al., 2005, Astrophys. J., 622, 759

Groth E. J., Peebles P. J. E., 1977, Astrophys. J. 217, 385

Jing Y. P., Börner G., 2004, Astrophys. J., 607, 140

Kulkarni G. V. et al., 2007, MNRAS, 378, 1196; arXiv:astro-ph/0703340

Kerscher M., Szapudi I., Szalay A. S., 2000, Astrophys. J. Lett., 535, L13; arXiv:astro-ph/9912088

Landy S. D., Szalay A. S., 1993, Astrophys. J., 412, 64.

Laurent P. et al., 2016, JCAP, 11, 060; arXiv:1602.09010

Marra V., Isidro E. G. C., 2019, MNRAS, 487, 3419, arXiv:1808.10695

Norberg P., Baugh C.M., Gaztaaga E., Croton D.J., 2009, MNRAS 396, 19; arXiv:0810.1885

Novaes C. P., Bernui A., Ferreira I. S., Wuensche C. A., 2014, JCAP, 01, 018; arXiv:1312.3293

Novaes C. P., Bernui A., Marques G. A., Ferreira I. S., 2016, MNRAS, 461, 1363; arXiv:1606.04075

Novaes C. P., Bernui A., Xavier H. S., Marques G. A., 2018, MNRAS, 478, 3253; arXiv: 1805.04078

Marques G. A., Novaes C. P., Bernui A., Ferreira I. S., 2018, MNRAS, 473, 165; arXiv: 1708.09793

Marques G. A., Bernui A., 2019; arXiv:1908.04854

McBride C. K. et al., 2010, Astrophys. J., 726, 13; arXiv:1012.3462

Marín F., 2011, Astrophys. J., 737, 97; arXiv:1011.4530

Marín F. A. et al., 2013, MNRAS, 432, 2654; arXiv:1303.6644

Materne J., 1988, Acta Cosmologica, 15, 49

Pâris I., et al., 2017, Astronomy \& Astrophysics, 597, A79.

Peacock J. A., 1999, Cosmological Physics, Cambridge Univ. Press

Peebles P. J. E., Groth, E. J., 1975, Astrophys. J., 196, 1 
Peebles P. J. E., 2001, ASP Conference Proceedings, 252, 201; arXiv:astro$\mathrm{ph} / 0103040$

Peebles P. J. E., Yu, J. T., 1970, Astrophys. J., 162, 815

Piattella O. F., 2018, Springer; arXiv: 1803.00070

Salazar-Albornoz S., Sánchez A. G., Padilla N. D., Baugh C. M., 2014, MNRAS, 443, 3612; arXiv: 1402.3590

Salazar-Albornoz S. et al., 2017, MNRAS, 468, 2938; arXiv:1607.03144

Sánchez E. et al., 2011, MNRAS, 411, 277; arXiv:1006.3226

Slepian Z. et al., 2017a, MNRAS, 468, 1070

Slepian Z. et al., 2017b, MNRAS, 469, 1738; arXiv:1607.06097

Springel V., Frenk C. S., White S. D. M., 2006; Nature 440, 1137; arXiv: astro-ph/0604561

Sunyaev R. A., Zel'dovich Y. B., 1970, Astrophys. Space Sci., 7, 3

Szapudi I., Szalay A. S., 1998, Astrophys. J. Lett., 494, L41

Xavier H. S., Abdalla F. B., Joachimi B., 2016, MNRAS, 459, 3693; arXiv: 1602.08503

Zheng Z., 2004, . The Astrophys. J., 614, 527; arXiv: astro-ph/0405527 